\title{
Applications and Perspectives of Biosensors for Diagnostics in Infectious Diseases
}

\author{
Renata Kelly Mendes ${ }^{1}$, Giovanna R Degasperi ${ }^{2}$, Frank Herman Behrens ${ }^{3}$, Larissa Marcelino ${ }^{4}$, Ahron Sicard ${ }^{2}$, Karina \\ Villalpando $^{5}$, Sérgio L Pinheiro ${ }^{4,5}$ and Augusto Etchegaray ${ }^{1,4 *}$
}

${ }^{1}$ Faculty of Chemistry, Pontifical Catholic University of Campinas, Brazil

${ }^{2}$ Immunology League, Faculty of Medicine, Pontifical Catholic University of Campinas, Brazil

${ }^{3}$ Faculty of Electric Engineering, Pontifical Catholic University of Campinas, Brazil

${ }^{4}$ Post-Graduation in Health Sciences, Pontifical Catholic University of Campinas, Brazil

${ }^{5}$ Dental School, Pontifical Catholic University of Campinas, Brazil

*Corresponding author: Augusto Etchegaray, Faculty of Chemistry, Post-Graduation in Health Sciences, Pontifical Catholic University of Campinas, Campinas, Brazil

Submission: 眥 December 21, 2017; Published: 望笽 January 12, 2018

\begin{abstract}
Infectious diseases are considered a major cause of morbidity and/or mortality worldwide, despite the development of preventive and control strategies. Infectious diseases result from the invasion of body tissues by disease-causing microorganisms. The occurrence and development of infectious diseases are closely associated with the functional state of the immune system. Interleukins play significant roles in modulating the immune response of the host during the course of an infectious disease, contributing to the maintenance of the homeostasis of the immune system. The use of biosensors for the detection of interleukins is a new and important strategy for the diagnosis of the infection and the immunological response profile that occurs for specific diseases, thus helping in the effective diagnosis, and allowing physicians to design treatment in a faster way, which contributes to better prognosis. For example, in the oral environment there are two major infectious diseases: caries and periodontitis. The microbes involved are respectively, Streptococcus mutans and Porphyromonasgingivalis. The presence of specific interleukins in saliva or crevicular fluid will indicate that there is potentially an infectious disease, which could be connected with either caries or periodontal disease. For instance, an indicative for caries could be the presence of IL- 6 in saliva, while periodontal disease would be confirmed by the presence of IL-17 in crevicular fluid. In addition, both diseases could be confirmed by the detection of higher levels of each of one of the pathogens. Considering the development of biosensor design and construction, the answer to all these question could be obtained in a single analysis.
\end{abstract}

\section{Immune Response Associated to Infection}

It is known that the consequences of an immune response against a microorganism are often closely correlated with the interleukins that are produced by cells of innate or adaptive immunity. In adaptive immunity, the production of interleukins by TCD4+ ou T-helper cellsis arranged according to the differentiation of these cells. They can differentiate into distinct subtypes characterized as Th1, Th2 e Th17, among others, each of which has specialized tasks considering the type of interleukin that is secreted [1]. The TCD4+cell differs in a particular subtype according to the microorganism involved in the infectious disease. Microorganisms, when captured by cells of innate immunity, induce the production of certain interleukins that will direct the polarization of the TCD4+cells. These interleukins induce the production of transcription factors that will determine specific signaling pathways, which are responsible for the production of interleukins for each of these $\mathrm{T}$ cell patterns [2,3].

Th1 is an important standard in the immune response to viruses, bacteria and parasites through the production of IL-2 and IFN- $\gamma$ cytokines and Th2 acts in the response especially against extracellular parasites as a function of the production of IL-4, IL-5 and IL-6 [4,5]. The first signs of independent Th1/Th2 polarization appeared in the mid-1990s, with the discovery of IL17.The TCD4+lymphocytes producing IL-17 were termed Th17. These cells play a key role in the control of the intestinal microbiota as well in the defense against bacteria and fungi. In vivo and in vitro studies have demonstrated that IL-17 can initiate or amplify various inflammatory pathways [6]. IL-17 is of particular interest in the pathogenesis of periodontal disease due to its role both in the inflammatory process associated with disease and in the protective function against the microorganism $P$. gingivalis. The mechanisms associated with IL-17 in this context include the ability not only to orchestrate recruitment of neutrophils to infection sites but also to stimulate the production of antimicrobial peptides such as defensins and cathelicidins [7]. The possibility of detecting interleukins at the site of infection is important in determining the type of immune response and thereby tracing forms of treatment and/or monitoring their effect. The detection of interleukins (IL- 
6) associated with the development of caries in children and the analysis of their progression can be monitored by immunological assays using IL-6 antibodies [8]. The same system can be adapted for biosensors [9]. The analysis can be carried out using saliva samples or crevicular fluid. In the latter case the area for collection material should be properly insulated and dried with sterile gauze. The supragingival portion of the bacterial biofilm should be removed previously, and then gingival fluid samples can be obtained by placing paper strips inside the periodontal sulcus for 30 seconds. The collected fluid can be analyzed by several methods, including biosensors [10,11].

\section{Biosensors}

Biosensors can be defined as analytical devices that produce signal after a biochemical reaction/interaction, using a biological molecule as recognition element [12]. Biosensors represent a promising tool to supplement sophisticated techniques, due to their unique characteristics, such as high selectivity, relative low cost, facility of automation and the possibility of data acquisition in real time [13].

However, for the device to be selective, the immobilization of the recognition element must be efficient. There are a number of methods used for a stable immobilization, but the most used procedures are performed by physical adsorption [14], gel occlusion [15], a microencapsulation [16] and covalent bond [17] or covalent crosslink [18]. All these methods present many variantsand the choice of the most appropriate depends oneach particular case, considering the type of desired application, format and stability of the final device.

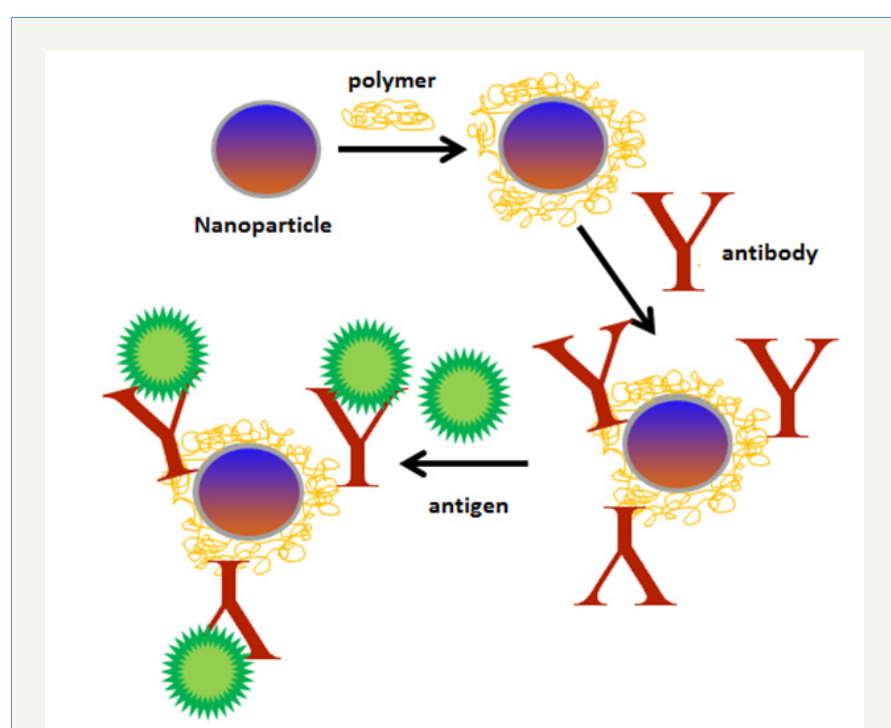

Figure 1: General scheme of immobilization using nanoparticles with a physically adsorbed polymer in which antibodies were covalently attached.

The most approaches involving immobilization of the recognition element on sensor surfaces include the use of nanotechnology. Nanomaterials in the construction of these devices offer unique features and are promising for faster responses and greater sensitivity than in planar surface configurations [19]. Due to its small size, the gain in surface area and strong adsorption properties of molecules and biomolecules has contributed to the successful use of nanomaterials on the development of biosensors, especially those with the possibility of miniaturization [20]. Figure 1 shows schematically an application of the use of nanomaterials for biomolecule immobilization on electrodic surface.

Recently, miniaturized and disposable biosensors have been the subject of great attention in the analyzes of chemicals and biological compounds. The success of these novel devices are based on the high performance in the determinations and versatility, given the size and format of the devices, the consequent minimization of reagent's consumption and low generation of residues, besides the main feature of the apparatus that is to the potential use in field analysis [21]. In addition, imprinted biosensors have a gain in sensitivity, since it refers to microscale, allowing lower detection limits to be achieved.

Adding to that, recent studies report the use of miniaturized electronic components and micro fabrication technologies for the construction of compact and fully integrated biosensors. For instance, the work of Derkus [22] reviews three different type of micro-engineered sensing devices, which are developed using micro/nano-patterning techniques, microfluidic technology, and micro-electro-mechanics system based technology (MEMS). The author discusses the necessary adaptation of current sensor technology to the human clinics. The proposed systems should deal with real biological samples such as urine, blood, cerebrospinal fluid, or cancerous tissue homogenate. Telemedicine based on the smart phone technology can also be used together with portable and home-use biosensors that may help to transmit clinical measurements through the telecommunication network to any place.

According to Pruna et al. [23] low-cost miniaturized potentiostats, with good measurement characteristics (e.g. bandwidth, determination of capacitive/inductive contribution to sensor's impedance and parallel screening) is still an unresolved challenge in bioelectronics. In their work, the combination of simple analogue circuitry together with powerful microcontrollers and a digital filter implementation is presented as an alternative to complex and incomplete architectures reported in the literature. A low-cost acquisition electronic system fully integrated with a biosensor's platform containing eight gold working microelectrodes and integrated reference and counter electrodes was developed and validated. The manufacturing cost of the prototype was kept below 300 USD. The performance of the proposed device was benchmarked against a commercial impedance analyzer through the electrochemical analysis of a highly sensitive biosensor casestudy. The results showed very promising for the development of low-cost, portable and miniaturized electrochemical systems for point-of-care and environmental diagnosis.

Ramfos et al. [24] presents the architecture and design of a compact, multichannel, hybrid-multiplexed potentiostat for 
performing electrochemical measurements on continuously biased electrode arrays. The proposed architecture utilizes a combination of sequential and parallel measurements, to enable high performance whilst keeping the system low-cost and compact. The accuracy of the signal readout is maintained by following a special multiplexing approach, which ensures the continuous biasing of all the working electrodes of an array. After sampling the results, a digital calibration technique cancels errors from component inaccuracies. A prototype printed circuit board (PCB) was designed and built using off-the-shelf components for the realtime measurement of the amperometric signal of 48 electrodes. The operation and performance of the PCB was evaluated and characterized through a wide range of testing conditions, where it exhibited high linearity (R2> 0.999) and a resolution of $400 \mathrm{pA}$.

Biosensors are able to make the appropriate interface with the electrochemical microelectrode assembly and the necessary electronic circuits for stimulus and voltage and current measurements according to the voltammetry and amperometry techniques. The sensor module is built in a similar way to those mentioned in the literature, being directly connected to the processor module, composed of a set of electronic circuits that performs the function of a potentiostat or galvanostat. The processor module also contains a microcontroller that performs the control of the various internal submodules and sequencing of the measurements, according to the previously defined parameters. It is also responsible for communication with the external medium via USB. The captured data is processed in a computer by an application software that allows the definition of control parameters of the processor module and the visualization of the measurement results in graphical or numerical form depending on the analysis performed.

The biosensor platform can be customized for different electrochemical analysis, with the definition of different measurement ranges and different sensitivities, being flexible to be adapted to different applications in clinical (or medical) analysis. Baraket et al. [9] developed a fully integrated electrochemical biosensor platform for interleukin detection at minute concentrations. The work describes a chip fabricated on silicon, having dual-side reference electrode (RE), a single central counter electrode (CE) between the REs, and 2 groups of four gold working microelectrodes (WEs) each side of the REs. Using eight gold Wes, the design increases the sensitivity of detection, decreases the time of measurements, and allows a simultaneous detection of various interleukin biomarkers.

Thus, with the advances in biosensor technology, it will be possible to analyze a number of biomarkers in field determinations, that is, it might be possible to test for biomarkers at dental clinics, at hospital and in emergency patient treatment, transport and removal to a safer place. Therefore, considering the dangers associated to infection, the design and applications of imprinted biosensors for quick determinations in many different types of situations, will find successful applications.

\section{References}

1. Yamane H, Paul WE (2013) Early signaling events that underlie fate decisions of naive CD4+ T cells towards distinct T-helper cell subsets. Immunol Rev 252(1): 12-23.

2. Zhu J, Yamane H, Cote-Sierra J, Guo L, Paul WE (2006) GATA-3 promotes Th2 responses through three different mechanisms: induction of Th2 cytokine production, selective growth of Th2 cells and inhibition of Th1 cell-specific factors.Cell Res 16(1): 3-10.

3. Pandiyan P, Zhu J (2015) Origin and functions of pro-inflammatory cytokine producing Foxp3+ regulatory T cells. Cytokine 76(1): 13-24.

4. Zhang Y, Zhang Y, Gu W, He L, Sun B (2014) Th1/Th2 cell's function in immune system. AdvExp Med Biol 841: 45-65.

5. Walker JA, McKenzie ANJ (2017) TH2 cell development and function. Nat Rev Immunol. doi: 10.1038/nri.2017.118.

6. Hoe E, Anderson J, Nathanielsz J, Toh ZQ Marimla R, et al. (2017) The contrasting roles of Th17 immunity in human health and disease. Microbiol Immunol 61(2): 49-56.

7. Zenobia C, Hajishengallis G (2015) Basicbiology and role of interleukin-17 in immunity and inflammation.Periodontol 2000 69(1): 142-159.

8. Menon MM, Balagopal RV, Sajitha K, Parvathy K, Sangeetha GB, et al. (2016) Evaluation of salivary interleukin-6 in children with early childhood caries after treatment. Contemporary Clin Dent 7(2): 198202.

9. Baraket A, Lee M, Nadia Zine N, Sigaud M, Bausells J, et al. (2017) A fully integrated electrochemical biosensor platform fabrication process for cytokines detection. Bios Bioelectron 93: 170-175.

10. Ambrósio LM, Rovai ES, França BN, Balzarini DA, Abreu IS, et al. (2017) Effects of periodontal treatment on primary sjögren's syndrome symptoms. Braz Oral Res 31: e8.

11. NazarMajeed Z, Philip K, Alabsi AM, Pushparajan S, Swaminathan D, et al. (2016) Dis Markers. Identification of Gingival Crevicular Fluid Sampling, Analytical Methods, and Oral Biomarkers for the Diagnosis and Monitoring of Periodontal Diseases: A Systematic Review. 2016:1804727.

12. Parikha M (2016) Biosensors and their applications - A review. J Oral Biol Craniofac Res 6(2): 1 153-159.

13. Roda A, Mirasoli M, Michelini E, Di Fusco M, Zangheri M (2016) Progress in chemicalluminescence-basedbiosensors: A criticalreview. Biosens Bioelectron 76: 1647-1679.

14. Tsai MC, Tsai YC (2012)Adsorption of glucose oxidase at platinummultiwalled carbon nanotube-alumina-coated silica nanocomposite for amperometric glucose biosensor. Sensor Actuat B 166-167: 802-808.

15. Chen X, Zhu J, Tian R, Yao C (2012) Bienzymatic glucose biosensor based on three dimensional macroporous ionic liquid doped sol-gel organicinorganic composite. Sensor Actuat B 163(1): 272-280.

16. Buiculescu R, Chaniotakis NA (2012) The stabilization of Au NPAChEnanocomposites by biosilica encapsulation for the development of a thiocholine biosensor. Bioelectrochem 86: 72-77.

17. Batra B, Lata S, Sunny, Rana JS, Pundir CS (2013) Construction of an amperometric bilirubin biosensor based on covalent immobilization of bilirubin oxidase onto zirconia coated silica nanoparticles/chitosan hybrid film. Biosens Bioelectron 44: 64-69.

18. Ruska N, Danka I, Janina V, Tzonka G (2010) New amperometric glucose biosensor based on cross-linking of glucose oxidase on silica gel/ multiwalled carbon nanotubes/polyacrylonitrile nanocomposite film. Sens Actuat B 148(1): 59-65.

19. Zhao W, Fang Y, Zhu Q, Wang K, Liu M, Huang X, et al. (2013) A novel glucose biosensor based on phosphonic acid-functionalized silica 
nanoparticles for sensitive detection of glucose in real samples. Electrochim Acta 89: 278-283.

20. Arya SK, Saha S, Ramirez-Vick JE, Gupta V, Bhansali S, et al. (2012) Recent advances in $\mathrm{ZnO}$ nanostructures and thin films for biosensor applications: Review. Anal Chim Acta 737: 1-21.

21. Siangproh W, Dungchai W, Rattanarat P, Chailapakul O (2011) Nanoparticle-based electrochemical detection in conventional and miniaturized systems and their bioanalytical applications: A review. Anal Chim Acta 690(1): 10-25.

Creative Commons Attribution 4.0

International License

For possible submission use the below is the URL
22. Derkus B (2016) Applying the miniaturization Technologies for biosensor design. Biosensors and Bioelectronics 70: 901-913.

23. Pruna R, Palacio F, Baraket A, Zine N, Streklas A, et al. (2018) A lowcost and miniaturized potentiostat for sensing of biomolecular species such as TNF- $\alpha$ by electrochemical impedance spectroscopy. Biosens Bioelectron 100: 533-540.

24. Ramfos I, Vassiliadis N, Blionas S, Efstathiou K, Fragoso A, et al. (2013) A compact hybrid-multiplexed potentiostat for real-time electrochemical biosensing applications. Biosens Bioelectron 47: 482-489,

\section{Your subsequent submission with Crimson Publishers}

\section{will attain the below benefits}

- High-level peer review and editorial services

- Freely accessible online immediately upon publication

- Authors retain the copyright to their work

- Licensing it under a Creative Commons license

- Visibility through different online platforms

- Global attainment for your research

- Article availability in different formats (Pdf, E-pub, Full Text)

- Endless customer service

- Reasonable Membership services

- Reprints availability upon request

- One step article tracking system 